\title{
Lernen aus der Analyse von Haftpflicht-Versicherungsfällen
}

Lediglich für den Bereich der Gutachterkommissionen und Schlichtungsstellen werden Arzthaftungsfälle systematisch erfasst und Ergebnisse durch die Ärztekammern veröffentlicht. Für 2010 waren dies 11016 Fälle, was ca. einem Viertel aller Behandlungsfehlervorwürfe entsprechen soll [1]. Das Fehlen einer Gesamtstatistik überrascht, denn bei einer Fehlerrate von rund 30\% kann Haftpflicht-Fällen eine sehr hohe Relevanz für die Fehleranalyse und -vermeidung zugerechnet werden.

Als einer der wenigen Leistungserbringer erfasst Helios Haftpflicht-Ansprüche pro Klinik und publiziert diese bis hin zur Darstellung von sogenannten „Never Events“ wie Seiten-/Verwechslungen und verbliebene Fremdkörper in seinen Jahresberichten (Tab. 1).

Tab. 1 Haftpflichtffälle bei HELIOS. Nach Melde-/Antragsjahr.

\begin{tabular}{|c|c|c|c|c|}
\hline & 2008 & 2009 & 2010 & $6 / 2011$ \\
\hline $\begin{array}{l}\text { Haftpflicht-Fälle } \\
\text { gesamt: }\end{array}$ & 634 & 731 & 645 & 272 \\
\hline $\begin{array}{l}\text { Haftpflicht-Fälle } \\
\text { Akut }\end{array}$ & 584 & 671 & 601 & 245 \\
\hline $\begin{array}{l}\text { Akutquote auf } \\
1000 \text { vollst. Fälle }\end{array}$ & 1,1 & 1,1 & 1,0 & 0,8 \\
\hline $\begin{array}{l}\text { Seiten-/ } \\
\text { Verwechslung }\end{array}$ & 4 & 4 & 5 & 3 \\
\hline Fremdkörper & 18 & 19 & 14 & 9 \\
\hline \multicolumn{5}{|l|}{$\begin{array}{l}\text { Schlichtungsstel- } \\
\text { lenverfahren: }\end{array}$} \\
\hline Anträge bei SST & 194 & 226 & 252 & 105 \\
\hline SST-Fehlerquote & $30,0 \%$ & $26,8 \%$ & $27,6 \%$ & $30,2 \%$ \\
\hline
\end{tabular}

Der Großteil der Ansprüche gegen Helios wird mittlerweile ohne Einbezug eines Versicherers durch einen zentralen Dienst bearbeitet. Neben besserer Kommunikation und schnellerer Klärung gegenüber dem Patienten ist eine verbesserte Analyse hinsichtlich Risiko- und Qualitätsmanagement-Maßnahmen das Ziel.

Alle Ansprüche werden in einer Datenbank nach verschiedenen Kategorien kodiert. Diese umfassen u.a. Vorwurf/Fehlerursache, juristische Bewertung und Finanzdaten. Seit der Ersterfassung im Jahr 2007 sind mehr als 3000 Ansprüche und haftungsrelevante Vorfälle aufgezeichnet. Dieses Behandlungsfehler-Register ermöglicht jederzeit spezifische Auswertungen. Quartalsweise erfolgt eine Berichterstattung mit Klinikvergleich. Ergänzend zum Qualitätsmanagement mit Routinedaten werden Haftpflicht-Fälle nun den medizinischen Fachgruppen für Peer Reviews oder Fall-Kasuistiken zur Verfügung gestellt. Zum Beispiel konnten aus der Analyse von „Never Events“ Schwachstellen beim Einsatz der OP-Checklisten identifiziert werden. Die Fachgruppe Pflege führte auf Basis von DekubitusFällen überhaupt erstmals ein zentrales Review durch.

\section{Haftpflichtverfahren als Indikator der Qualitätsmessung?}

Wie Critical Incident Reporting (CIRS), Patient Safety Indicator (PSI) oder Qualitätssicherung mit Routinedaten (QSR)/Initiative Qualitätsmedizin (IQM) stellt auch ein Haftpflicht-Register eine Methode der Qualitätsmessung dar. Weil Ansprüche sowieso bearbeitet werden müssen, entfällt ein zusätzlicher Erhebungsaufwand weitgehend. Die einzelnen Anspruchszahlen mögen gering sein, deren Erkenntnis- und Mehrwert ist jedoch bemerkenswert. Zunächst geben die im Zuge der Klärung der Haftungsfrage vorliegenden medizinischen Gutachten und Stellungnahmen oft einen einzigartigen Einblick in Fehlerursachen und im Sinne des „Aus Fehlern Lernens“. Zudem werden bei Haftpflichtverfahren eine ganze Reihe an Ereignissen bekannt, die in Routinedaten bisher nicht umfasst sind, z.B. der Anästhesie oder Handchirurgie. Aber auch bei gut abgebildeten Behandlungen wie der Orthopädie/Unfallchirurgie - woraus ein Großteil der Haftpflichtverfahren resultiert - werden Probleme durch die Rückspiegelung der Ergebnisse nach dem stationären Aufenthalt oft erst direkt nachvollziehbar. Inwieweit Haftpflicht als Querschnittsindikator statistischen Anforderungen genügen kann - etwa als Maß für Patientensicherheit - müsste Gegenstand der weiteren Versorgungsforschung sein. In den USA sind jedenfalls umfangreiche Haftpflicht-BenchmarkReports inzwischen etabliert [2].

Autorenerklärung: Es bestehen keine finanziellen Interessenkonflikte in Zusammenhang mit diesem Artikel.

\section{Literatur}

1 Bundesärztekammer. Statistische Erhebung der Gutachterkommissionen und Schlichtungsstellen für das Statistikjahr 2010. Berlin: 2011

2 Ruoff G, MPH, CPHRM. (Ed.). Annual Benchmarking Report - Malpractice Risks in Obstetrics. CRICO Strategies, 2010. Cambridge: 2011
A. Meyer

Qualitätsmanagement

Schlüsselwörter

Behandlungsfehlerregister

Patientensicherheit

Qualitätsmessung

Keywords

medical malpractice register

$\checkmark$ patient safety

quality measurement

quality measurement

Institut

HELIOS Kliniken GmbH, Berlin

Bibliografie

DOI $10.1055 / \mathrm{s}-0031-1286084$

Dtsch Med Wochenschr 2011 ;

136: S55 - (c) Georg Thieme

Verlag KG Stuttgart · New York . ISSN 0012-0472

Korrespondenz Andreas Meyer HELIOS Kliniken GmbH Friedrichstraße 136 10117 Berlin Tel. 030/521321-451 eMail andreas.meyer@ helios-kliniken.de 